\title{
On buckyonions as a carrier of the $2175 \AA$ interstellar extinction feature
}

\author{
Juhua Chen ${ }^{1,2}$, Moping $\mathbf{L i}^{2}$, Aigen $\mathbf{L i}^{2}$, and Yongjiu Wang ${ }^{1}$ \\ ${ }^{1}$ Department of Physics, Hunan Normal University, Changsha, Hunan 410081, China \\ email: jhchen@hunnu.edu.cn, wyj@hunnu.edu.cn \\ ${ }^{2}$ Department of Physics \& Astronomy, University of Missouri, Columbia, MO 65211, USA \\ email: limo@missouri.edu, lia@missouri.edu
}

\begin{abstract}
In recent years buckyonions have been suggested as a carrier of the $2175 \AA$ interstellar extinction feature, based on the close similarity between the electronic transition spectra of buckyonions and the $2175 \AA$ interstellar extinction feature. We examine this hypothesis by calculating the interstellar extinction with buckyonions as a dust component. It is found that dust models containing buckyonions (in addition to amorphous silicates, PAHs, graphite or amorphous carbon) can closely reproduce the observed interstellar extinction curve. However, a more severe challenge to the buckyonion hypothesis is provided by the non-detection of the $\sim 7-8 \mu \mathrm{m} \mathrm{C}-\mathrm{H}$ stretching bands expected from buckyonions in the diffuse interstellar medium. This will allow us to place an upper limit on the abundance of buckyonions.
\end{abstract}

Keywords. Dust, extinction, ISM: lines and bands, ISM: molecules

\section{Introduction}

In the interstellar medium (ISM), the strongest spectroscopic extinction feature is the $2175 \AA$ bump. Since Stecher \& Donn (1965) first detected this ultraviolet (UV) extinction feature through rocket observations, the origin of this feature and the nature of its carrier(s) are still an enigma. Many candidate materials, including graphite, amorphous carbon, graphitized (dehydrogenated) hydrogenated amorphous carbon, nano-sized hydrogenated amorphous carbon, quenched carbonaceous composite, coals, $\mathrm{PAHs}$, and $\mathrm{OH}^{-}$ ion in low-coordination sites on or within silicate grains have been proposed, while no single one is generally accepted (see Li \& Greenberg 2003 for a review).

Recently, Ruiz et al. (2005) theoretically simulated the UV/optical photo-absorption spectra of buckyonions. They found that the calculated absorption spectra of buckyonions alone almost perfectly fit the $2175 \AA$ interstellar extinction feature. Therefore, they proposed buckyonions as its carrier.

However, it is well recognized that in the ISM, in addition to the $2175 \AA$ extinction carrier, there must exist other dust components as well - there must be a population of amorphous silicate dust, as indicated by the strong, ubiquitous 9.7 and $18 \mu \mathrm{m}$ interstellar absorption features; there must be a population of aromatic hydrocarbon dust (presumably polycyclic aromatic hydrocarbon $[\mathrm{PAH}]$ molecules), as indicated by the distinctive set of "unidentified" infrared (UIR) emission bands at 3.3, 6.2, 7.7, 8.6, and $11.3 \mu \mathrm{m}$ ubiquitously seen in the ISM; there must also exist a population of aliphatic hydrocarbon dust, as indicated by the $3.4 \mu \mathrm{m} \mathrm{C}-\mathrm{H}$ absorption feature which is also ubiquitously seen in the diffuse ISM of the Milky Way and external galaxies.

Although buckyonions are able to closely reproduce the $2175 \AA$ extinction feature, it is not clear if dust models consisting of buckyonions, amorphous silicates, PAHs, and other carbon dust species (amorphous carbon, hydrogenated amorphous carbon, or graphite) are capable of fitting the $2175 \AA$ extinction feature since one may intuitively expect the 
almost perfect fit by buckyonions would easily be distorted by the addition of other dust components. It is the purpose of this short report to examine this issue.

\section{Extinction of Multi-component Dust Models}

We consider dust models consisting of amorphous silicate dust, graphite, PAHs, and buckyonions. We take the size distributions of silicate and graphite dust to be that of Weingartner \& Draine (2001; hereafter WD). The quantity of silicate dust is taken to be consistent with the interstellar depletion: we assume that all cosmically available Si, $\mathrm{Mg}$, and $\mathrm{Fe}$ are locked up in amorphous silicate dust. We assume the interstellar Si and $\mathrm{C}$ abundances (relative to $\mathrm{H}$ ) to be solar $(\mathrm{Si} / \mathrm{H}=35 \mathrm{ppm}, \mathrm{C} / \mathrm{H}=355 \mathrm{ppm}$ ). With $\mathrm{C} / \mathrm{H}=140 \mathrm{ppm}$ in the gas phase, we have $\mathrm{C} / \mathrm{H}=215 \mathrm{ppm}$ left for carbon dust: buckyonions, PAHs, and graphite. The "UIR" bands require $\mathrm{C} / \mathrm{H} \approx 60 \mathrm{ppm}$ to be in PAHs (Li \& Draine 2001). So we only have $\mathrm{C} / \mathrm{H}=155 \mathrm{ppm}$ for buckyonions and graphite. As shown in Figure 1, with $\mathrm{C} / \mathrm{H}=20 \mathrm{ppm}$ in graphite and $\mathrm{C} / \mathrm{H}=130 \mathrm{ppm}$ in bukyonions (for which the absorption spectrum is taken from Ruiz et al. 2005), the silicate-graphitePAH-buckyonion model provides an excellent match to the interstellar extinction curve at $\lambda^{-1} \sim 3.5-7 \mu \mathrm{m}^{-1}$ (at present the photo-absorption spectra of buckyonions are available only in this wavelength range).

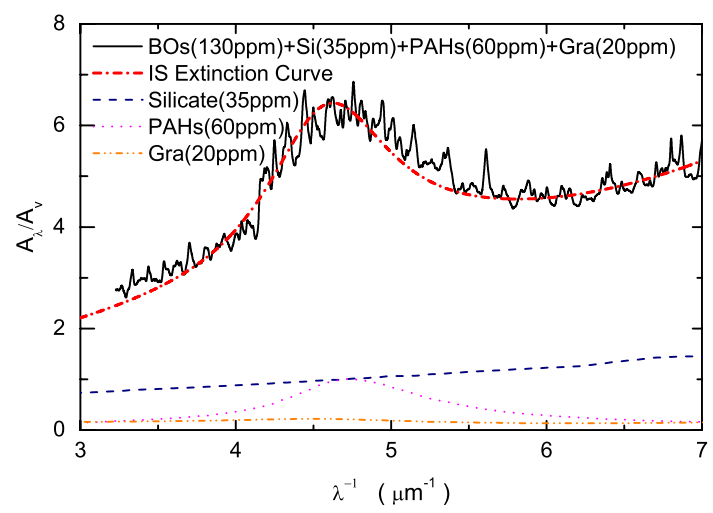

Figure 1. Comparison of the interstellar extinction (dot-dashed line) with the model extinction (solid line) obtained by summing up the contributions of buckyonions $(\mathrm{C} / \mathrm{H}=130 \mathrm{ppm})$, PAHs $(\mathrm{C} / \mathrm{H}=60 \mathrm{ppm})$, graphite $(\mathrm{C} / \mathrm{H}=20 \mathrm{ppm})$, and amorphous silicate dust $(\mathrm{Si} / \mathrm{H}=35 \mathrm{ppm})$.

\section{Future Work}

A powerful test of the buckyonions hypothesis would be in the IR. In the ISM, buckyonions (with their surface hydrogenated) will be stochastically heated by single UV photons and emit at the $\sim 7-8 \mu \mathrm{m} \mathrm{C}-\mathrm{H}$ stretching bands. The non-detection of these bands will allow us to place an upper limit on the abundance of buckyonions. Moreover, it would be interesting to see if the silicate-graphite-PAH-buckyonion model is able to reproduce the $\sim 2-3000 \mu \mathrm{m}$ overall IR emission of the Galactic ISM.

\section{Acknowledgements}

This project is supported by Hunan Provincial Natural Science Foundation of China.

\section{References}

Li, A. \& Draine, B. T. 2001, ApJ, 554, 778

Li, A. \& Greenberg, J. M. 2003, Solid State Astrochemistry, p. 37

Ruiz, A., Bretón, J., \& Gomez Llorente, J. M. 2005, Phys. Rev. Lett., 94, 105501

Stecher, T. P. \& Donn, B. 1965, ApJ, 142, 1681

Weingartner, J. C. \& Draine, B. T. 2001, ApJ, 548, 296 (WD) 